\title{
EXPERIMENTAL STUDIES OF THE BEAM-BREAKUP MODE ON ETA: COMPARISON WITH THEORY*
}

\author{
G. J. Caporaso and K. W. Struve \\ January 29,1982 \\ Lawrence Livermore National Laboratory \\ University of California \\ Livermore, CA 94550
}

\section{ABSTRACT}

The beam breakup mode has been observed and measured on ETA. Comparison between the measurements and the results of a computer code indicate that the beam breakup instability will be the most important limitation on current transport thru ATA. ETA Experiments that will enable a more accurate determination of the magnitude of the instability on ATA are discussed.

UCID- - 19402

DE22 017117

\footnotetext{
*The Lawrence Livermore National Laboratory is operated by the University of California for the U. S. Department of Energy under Contract No. H-7405-Eng-48.

This work is performed by LLNL for the U. S. Department of Defense under DARPA (DOD) ARPA Order No. 4395 Amendment No. 1, monitored by NSWC under document number N60921-82-WR-W0056.
}

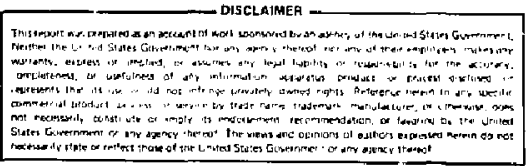




\section{Introduction}

In many linear accelerators the maximum achievable beam current is limited by the beam breakup (BBU) instability. This instability arises from a beam interaction with resonant modes of the accelerating cavities which have a strong transverse component of magnetic field at the beam position. Far the ETA and ATA accelerators the most important are the $\mathrm{TM}_{1 \text { no }}$ modes with only the $\mathrm{TM}_{130}$ mode being observed experimentally. The rf oscillations in each accelerator casity give the beam a transverse "kick" as it passes each cavity. The size of the displacement has been shown to grow as a power series in pulse width wt, the transverse impedance of the cavity $Z_{\perp} / Q$, and the beam current $I$. The highest order of the power series is the number of accelerator cavities $N$. For large $N$ the growth approaches an exponential form. There is also a damping effect which is strongly dependent on $Q(=\omega / \Delta \omega)$, and is of the form $\exp (-\alpha t)$, where $\alpha=\omega / 2 Q$. By reducing the $Q$ of the mode at $c$ an be made large enough to reduce the growth of the instability.

In this paper experimental observations of the beam breakup mode on the ETA are given. The experimental results are compared with code results in an effort to verify the physical model and associated assumptions used in the code. The goal is to understand BBU on the ETA so that we can somewhat more confidently predict its growth on the ATA. Results from code runs for the ATA are also given. However, these are subject to large uncertainties in some of the crucial input parameters. With this in mind the last section of the report is devoted to a discussion of planned experiments which are designed to address these uncertainties.

\section{The Experiment}

Experimental studies of the BBU mode have advanced on two fronts. The first efforts were directed at measuring both the $Q$ and $Z_{\downarrow} / Q$ of the cavity modes. 1 Then Bir ${ }^{2}$ showed that the $Q^{\prime}$ 's could be greatly reduced by placing ferrite on the drive blades and "floor" of the cavity. Later it was 
found that the Q's could be further reduced by placing a reflector in the corner of the accelerator cavity. The reflector serves to better transmit resonant modes through the ceramic insulator into the oil-filled regions of the cavity where the damping ierrite is located.

These changes to the accelerator cavity are shown in Figure 1. Aithough already at least partialiy reported, ${ }^{3}$ a summary of the cavity Q's and transverse impedances for the ETA and ATA cavities is given in lable 1 . The transverse impedances given are the values measured by Birx on the ETA cavity and corrected for transit time effects. ${ }^{4}$ Although not measured, it is expected that the transverse impedances of the ATA cavity do not significantly differ from those of the ETA cavity.

TABLE I

Sumary of Accelerator Cavity Q's and Transverse Impedances

\begin{tabular}{|c|c|c|c|c|c|c|}
\hline \multirow[b]{2}{*}{ Mode } & \multirow{2}{*}{$\begin{array}{l}\text { Frequency } \\
(\mathrm{MHz})\end{array}$} & \multirow[b]{2}{*}{$z_{\perp} / 0$ (ohms) } & \multicolumn{2}{|c|}{ ETA Q's } & \multicolumn{2}{|c|}{ ATA Q's } \\
\hline & & & Undamped & Damped & Undamped & Damped \\
\hline$T_{110}$ & 345 & 8.2 & 40 & * & - & * \\
\hline$T M_{120}$ & 605 & 8.1 & Ju & * & - & * \\
\hline$T M_{130}$ & $830+$ & 8.0 & 41 & 7 & 34 & 4 \\
\hline
\end{tabular}

In addition to tire cavity measurements, the BBU mode was aiso studied on the ETA accelerator. In late 1980, transverse oscillations of the beam at $830 \mathrm{MHz}$ were first observed. These were identified with the $\mathrm{TM}_{130}$ mode of the acceiel sting cavity. Furthermore, they were found to grow in amplitude through the accelerator, and to be very sensitive to beam current amplitude and risetime. In May 1981, the last two accelerator cavities of the ETA were modified as shown in Figure 1. In June the first two cavities were modified, and in August the remaining four cavities were modified. These modifications reduced the BBU oscillations considerably. The results of these experiments are shown later. 
The physical layout of the ETA accelerator section is shown in Figure 2. Shown are the eight accelerator cavities, the various solenoidal focusing magnets, the wall current monitors (also called "beam bugs"), and the location of the $r f B_{\theta}$ pickup loops. The initial experiments were done with roops placed in the vacuum pumpout stations. These are labeled RFA, B, and $C$ in the figure. Later experiments were done with sets of four probes at the positions of bean bugs 2, 4 and 5 . The change was made to avoid spurious resonances of the vacuum pumpout boxes, and to provide a geometry from which beam displacement could be easily determined.

A sketch of the rf loops used at the beam bug locations is shown in Figure 3 . With the first measurements the same probe was inserted in the top of the vacuum pumpout box through a wilson seal. The signal produced by the probe is a sum of contributions from the $\mathrm{dI} / \mathrm{dt}$ of the beam and the $\mathrm{dr} / \mathrm{dt}$ of the beam position. With a sinusoidal variation in the beam position $r_{b}$ $=r+\Delta r \sin \omega t$,

$$
V_{\text {probe }}=k \frac{d}{d t}\left(\frac{I}{r_{b}}\right) \cong \frac{k}{r}\left(i-I_{\omega} \frac{\Delta r}{r} \cos \omega t\right)=V_{I}+V_{r f}
$$

where $k=\mu_{0} A \delta / 2 \pi$, and $\delta$ is an empirically determined calibration factor of order 1/2. A is the loop cross sectional area. Both the dI/dt and $\mathrm{dr}_{\mathrm{b}} / \mathrm{dt}$ effects are easily seen in a typical signal. The large spikes near the beginning and end of the signal come from the rising and falling edges of the beam current. The oscillations in between, where $\mathrm{dI} / \mathrm{dt}$ is small, come from the transverse motion of the beam centroid. For ETA geometries, with $r=7.3 \mathrm{~cm}$ and $\delta=.55$, the beam displacement $\Delta r$ is then reiated to the $r f$ signal by

$$
\Delta r(\mathrm{~cm})=.0134 \frac{v_{r f}(\text { volts })}{I(\mathrm{kA}) f(\mathrm{GHz})} .
$$

The beam bugs, the other essential diagnostics, measure the electron beam current and centroid position versus time. ${ }^{5}$ The beam bug is a resictive section of thr beam tube wall across which the voltage drop from the return 
currents in the wall are measured. The total net current is determined from the sum of the voltages from four pickup ports which are separated $90^{\circ}$ from each other. The bean centroid position, on the other hand, is found by taking differences between signais from opposing ports. This is done in both the vertical ( $Y$ ) and horizontal $(X)$ directions. The bean offset in the $(X)$ direction for example is

$$
\Delta x(t)=\frac{v_{x}(t)}{2 V_{I}(t)}
$$

where $V_{X}=V_{\text {difference }} / 2$ and $V_{I}=V_{5 u m} / 4$. The $V_{I}, V_{X}$, and $V_{Y}$ signals are those shown with the following data.

For direct comparison with theory it is also necessary to know the beam energy throughout the accelerator. The beam energy is determined from a sum signal constructed fron voltage divider signals from each accelerating gap. This signal is periodically checked against an energy analyzer similar to the one used on the Astron experiments. ${ }^{6}$

A final parameter needed is the magnitude of the solenoidal $B_{Z}$ fjeld throughout the accelerator. The $B_{z}$ field strength is determined by calculating the on-axis field of each coil and suming the contributions from all the coils. The field contribution for each coil is easily determined from the geometrical shape of the coil and its DC current which is read from a meter. Field strengths determined in this manner are found to be within $5 \%$ of measured values.

\section{Experimental Technique}

The data from the $B_{\theta}$ loops was collected by two separate techniques. The first was to use a spectrum analyzer (SA) to display the frequency variation of the loop signa?. This is done by sending the signal through the $S A$ as it is slowly scanned in frequency. The output for any particular pulse is the $\mathrm{rf}$ amplitude within a $3 \mathrm{MHz}$ bandwidth of the scan frequency at the time 
of the pulse. As the machine continues to fire the scan frequency slowly changes and the amplitudes of the other frequencies are displayed. After many pulses of the accelerator all the frequencies within the scan range are covered. The result is an accumulation (not average) of the amplitudes of all the frequencies. The data is accumulated by taking an open shutter photograph of the spectrum analyzer CRT over a 10-minute period. To measure BBU mode gain it is necessary to repeat the process with the probes at the other locations. The difficulty with this technique is that the resulting signal includes pulse-to-pulse variations and that large amounts of beam time are neesled to record all the probe signals. An example of the type of data obtairied by this technique is shown in reference 3 .

The second technique that has beer: used and which is reported in this paper is to display the $B_{\theta}$ probe signals from all locations simultaneously using $1 \mathrm{GHz}$ bandwidth oscilloscopes (Tektronix 7104 with $7 \mathrm{~A} 29$ and $7 \mathrm{~B} 10$ plug-ins). This data along with simultaneous beam bug data provides a complete characterization of each shot. In this manner pulse-to-pulse variations in beam profile and initial offset can be treated.

\section{The Data}

A7though many simulianeous shots were taken, only the data from three representative shots is presented. Figure 4 shows the beam bug current and offset data for an $8 \mathrm{kA}$ shot for which on ly two accelerator cells were damped. The corresponding rf loop signal is shown in Figure $5 \mathrm{a}$. Data for two $10 \mathrm{kA}$ shots, with all accelerator cells damped, are given in Figures 6 and 7 . It is most interesting that with very similar beam currents and beam offsets the rf amplitudes are so considerably different. The amplitude difference arises from almost imperceptible differences in the beam current profiles Detiveen the two shots. All three of these shots are compared with code runs in the next section.

A summary of the BBI displacement $\wedge r$ versus the number of damped cavities for two current levels is given in Figure 8 . The quanticy ir is the transverse peak displacement from the beam axis. The spreas in points for 
each set of experiments is due to the pulse-to-pulse jitter in the initial beam conditions. The pulse shape also changed slightly between sets of experiments. Thereforo, the data can only be used to indicate a trend. Shots with $10 \mathrm{kA}$ are only shown with the four and eight damped cavity cases. Prior to having four damped cavities, $10 \mathrm{kA}$ operation of ETA had not been achieved. The larger rf amplitudes make transport of the beam through the accelerator much more difficult and presumably prevented the higher curre: leveis.

\section{The Beam Breakup Code}

The BBU code employs the single mode theory of Neil, Hall and Cooper to calculate the growth of the instability. ${ }^{7}$ Each accelerating cavity is characterized by three parameters; the angular frequency of the dominant mode $\omega$, its quality factor $Q$ and its transverse shunt impedance $Z_{\mathcal{L}} / Q$. These three quantities may be obtajned from bench tests.

The code utilizes solenoidal transport which is able to incorporate the accelerator "tune" or actual axial variation of che magnetic field throughout the accelerator. In the calculation the accelerator is divided into small $(-4 \mathrm{~cm})$ cells characterized by a constant magnetic field. The actual transport from cell to cell is represented by a $4 \times 4$ matrix which approximately treats the effects of fringing fields. 8

The measured tenipcrai profiles of beam current and displacement in the $X$ and $Y$ directions at the end of the injector are input as initjal conditions.

To compare the computed results with experiments the code calculates the voltage waveforms that the rf loops at various positions down the accelerator should produce.

Perhaps the most questionable assumption implicit in the formalism of the code is that of a single mode cavity. Although the frequency of the observed instability does correspond to that of a particular mode $\left(\mathrm{TM}_{130}\right)$ it is almost certain that the response of the cavity at that frequency is somewhat 
influenced by the adjacent modes. This is especially true in the present ETA configuration where cavity $Q$ 's are about 7 . This effect can be treated in the code by using an effective $z_{\perp} / Q$ which accounts for the influence of adjacent modes at the frequency in question.

\section{Comparison of Code Results With Data}

An experiment on a primarily undamped accelerator was simuiated. The first six cavities were undamped with $Q^{\prime}$ s of 41 . The last two cavities were damped with Q's of 7 . The observed mode frequency was $830 \mathrm{MHz}$. A Z $/ Q$ of 8 ohms was used. The input current and displacement profiles (not unfolded) are shown in Figure 4. The peak current for this ejperiment was about $8 \mathrm{kA}$. Figure 5 a shows a rf loop signal at the end of the accelerator. The corresponding code result is shown in Figure $5 \mathrm{~b}$.

To test the sensitivity of the results to the value of $Z_{\perp} / Q$ calculations were run witii yalues of $z_{\perp} / Q$ between 7 and 12 ohms. The results are shown in Figure 9.

Next, a sequential pair of shots at $10 \mathrm{kA}$ on a fully damped accelerator was simulated. The input displacement and current profiles for the first of these along with the $4 \mathrm{rf}$ loop output signals are shown in Figure 6 .

The corresponding data for the second shot is shown in Figure 7. It was found that a $Z_{\perp} / Q$ of 8 ohms did not give good agreenent with the data. Several values of $Z_{1} / Q$ were used. The results are shown in Table II. The observed ratio in amplitude of the instability for the two experiments is roughly 4. As $Z_{\perp} / Q$ is increased from 8 to 12 ohms, not only do the individual values approach the experimental ones but, the ratio from one exper iment to the other increases from 2 at $Z_{\perp} / Q=8$ ohms to roughly 3 at $\vec{z}_{\perp} / 0=12$ ohms. 
TABLE [ ]

\section{Experiment \#2}

$\begin{array}{lcc}\mathrm{Z}_{1} / 0 & \text { Code Results } & \text { Experiment } \\ 8 & 150 \mathrm{mv} & \\ 10 & 370 \mathrm{mv} & 1,000 \mathrm{mv}(.39 \mathrm{~cm}) \\ 12 & 850 \mathrm{mv} & \end{array}$

Experiment \#3

$2_{1} / 9 \quad$ Code Results Experiment

$8 \quad 75 \mathrm{mv}$

$102 \quad 150 \mathrm{mv} \quad 250 \mathrm{mv}(.1 \mathrm{~cm})$

This increase in effectivf $Z_{1} / Q$ from 8 for an undamped accelerator to 12 for a fully damped set of cavities may be due to the influence or overlapping modes as mentioned previously. It may also be a misinterpretation due to large uncertainties in the actual initial or iditions. That is, if the actual beam excitation of the $88 \mathrm{U}$ mode is larger than suspected, then a larger effective $Z_{\perp} / Q$ would be needed in order to match the data.

\section{Implications for ATA}

The possible range of expected BBU amplitudes at the end of ATA is showr. in Figure 10 for different operating conditions. The results of individual code runs are marked by solid circles. The solid lines are the result of an asymptotic formula valid for large numbers of cavities:

$$
\xi-\xi_{0} \frac{e}{2 \sqrt{2 \pi} \cdot(2 \Gamma)^{\frac{1}{4}}}
$$

where

$$
\Gamma=1.98 \times 10^{-12} \frac{N \omega}{B_{k G}}\left(\frac{2}{Q}\right) Q \frac{I}{17 \frac{}{k A}}
$$


and $\xi_{0}$ is the initial rf amplitude at the first cavity

$$
0=\frac{1.89 \times 10^{-3} \mathrm{~d}_{0}}{\mathrm{G}_{\mathrm{KG}}{ }^{\mathrm{T}} \mathrm{nS}}\left(\frac{\mathrm{I}_{\perp}}{\mathrm{Q}}\right) \frac{\mathrm{T}}{17} \overline{\mathrm{kA}} .
$$

Here $N$ is the number of cavities (150 for ATA), $d_{0}$ the initial bean offset in $\mathrm{cm}, \mathrm{T}$ the beam risetime in $\mathrm{ns}$ and $Z_{1} / Q$ the transverse shunt impedance in ohms. For Figure 10, $\mathrm{\tau}=10 \mathrm{~ns}$ and $\mathrm{d}_{0} \stackrel{1}{=} .1 \mathrm{~cm}$. The peak $\mathrm{rf}$ amplitude occurs some 20 to 30 ns into the pulse so that the precise pulse iength does not enter into the growth expression.

Examination of $F$ igure 10 reveals several facts. First, given the present uncertainty in $z_{\perp} 10$ for a fully damped cavity, the $10 \mathrm{kA}$ performance of ATA could be severely affected by beam breakup. Second, there is an enormous variation in beam breakup amplitude with relatively small changes in the critical parameters of $I, N, Z_{2} / Q$ and $Q$ and $B$ so that the uncertainty in $Z_{1} / Q$ spans the range from marginal performance to severely affected performance.

Other than the model uncertainties aiready discussed, there is the questionable assumption (for ATA) that the cavities are uncoupled from each other.

Aside from questions concerning physics assumptions implicit in the code there are potential difficulties in the present code-experiment comparison scheme. These ch.jefly involve the high sensitivity of the results to the precise shape of the current temporal profile and initial displacement profiles. Physically, the first accelerator cavity will respond to the Fourier corronent of the initial dipole moment (current times displacement) at the mode frequency. The first cavity produces a small rf output from this drive which will subsequently be amplified by the renaining cavities. In the code this initial rf output is very sensitive ta the details of the current and displacement. This uncertainty can be reilieved to a large extent by the tickler series of experiments to be discussed shortly. 
Finally, one further source of inaccuracy should be mentioned. Transport of current through the accelerator is not perfect; some loss of current occurs on almost all shots. The effect of the current loss is to reduce the growth of the BBU amplitude. The code has no privision for computing current loss so that experiments in which current loss was minimal must be selected for analysis.

\section{Experiments in Progress}

To address the concern over initial conditions, a tickler experiment was proposed. An offset, tunable rectangular cavity is to be insertad into the ETA beamline upstream of the accelerator. The beam self excites the ticklerr cavity and produces BBU oscillations on the beam which are detected at a set of $4 \mathrm{rf}$ loops spaced approximately a half cyclotron waveiength downstream of the tickler. An additional set of $4 \mathrm{rf}$ loops is in place at the end of the accelerator. The experiment is shown schematica? iy in Figure 11. In this mode of operation, the $r f$ gain of the acceierator can be measured and compared with code calculations. The tunability of the tickler should permit measurement of gain vs frequency over a several-hundred megahertz band. It is hoped that this information will facilitate a more accurate determination of $Z_{1} / Q$ and $Q$. 


\section{References}

1. D. L. Birx, Lawrence Livermore National Laboratory Report UCIO-18582, 1980.

2. D. L. Birx, Lawrence Livermaie National Laboratory Report UCID-18630, 1980.

3. R. J. Briggs, et a?, Proc. IEEE Acc. Conf., March 198?; and Lawrence Livermore National Laboratory Report UCRL-85650, 1981.

4. G. J. Caporaso, ATA Note 167 (1982).

5. T. J. Fessenden, B. W. Stallard, G. G. Berg, Rev. Sci. Instrum. 43, 1789 (1972).

6. T. J. Fessenden, Rev. Sci. Instrum. 43, 1090 (1972).

7. V. K. Neil, L. S. Hall, R. K. Cooper, Particle Accelerators 9, $2 T 3$ (1979).

8. D. N. Edwards, B. Rose, Nuci. Instrum. Meth. 7, 135 (1900). 


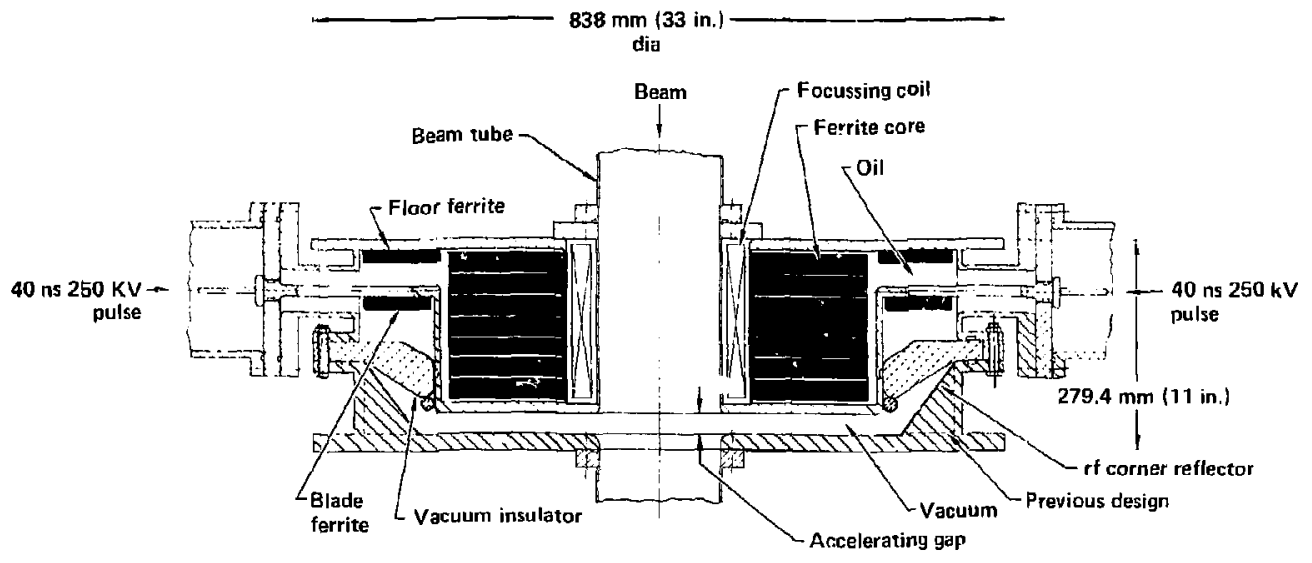

Figure 7. The ETA accelerating cevity modified to darnp the beam breakup instebility. In the ETA version, the ferrite if dampeners are placed only on the drive blades and not the floor, as shown. In the ATA version, the additianal ferrite is placed in both locations. 


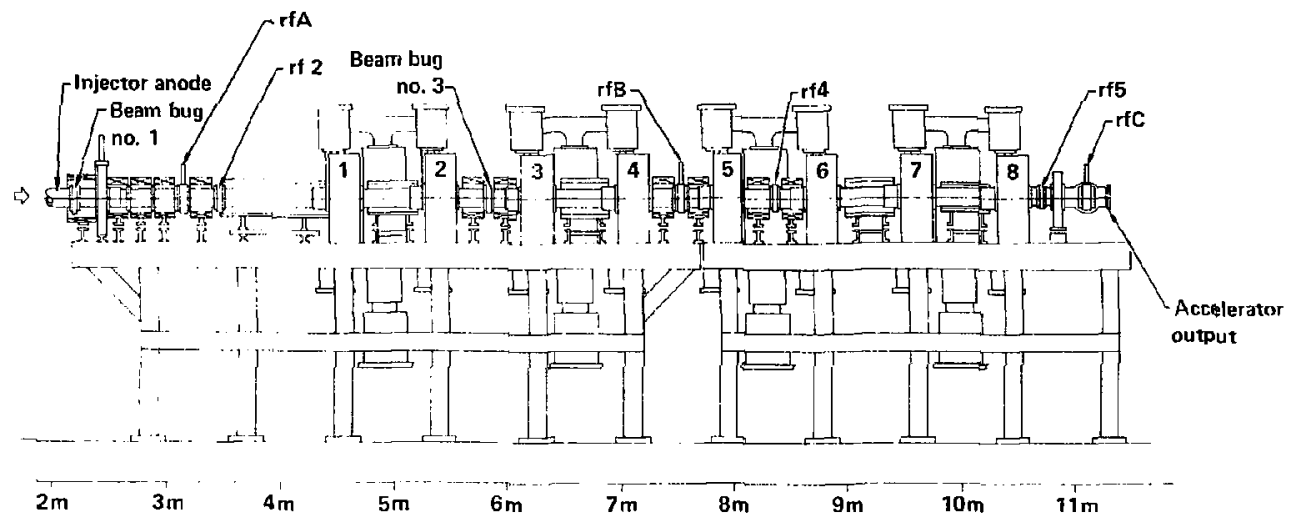

Figure ?. The ETA accelerator with beam bug and $B_{f}$ loop locations. The accelerator cavities, numbered 1 through 8 , were modified as shown in Figure 1, in 1981. Cavities 7 and 8 were modified in April, cavities 1 and 2 in June, and 3, 4,5 and 6 in August. 

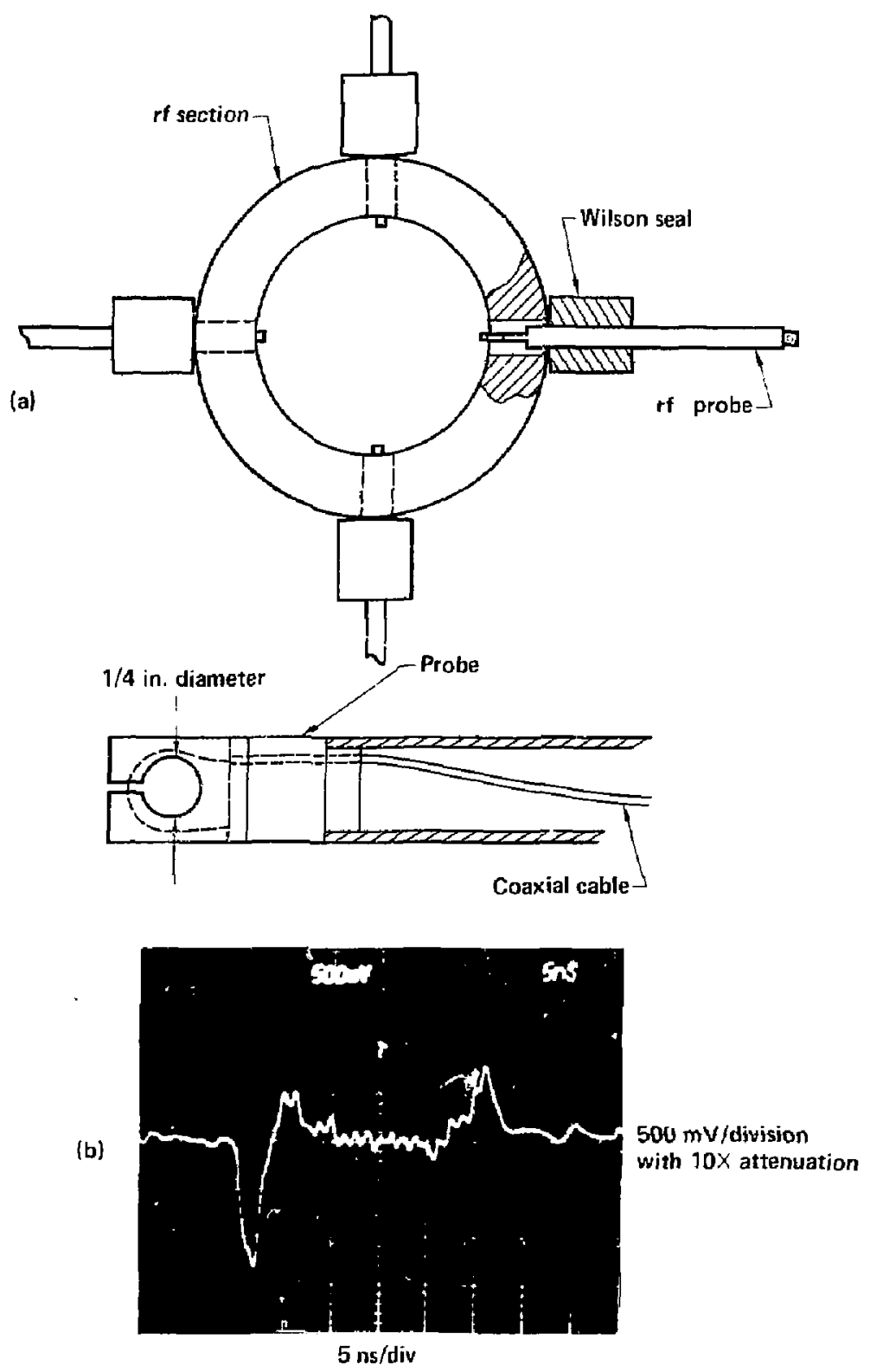

Figure 3. (a) The if pickup loops used for $B_{1}$ measurements of the beam break-up modes (b) A typical signal from the ETA. 


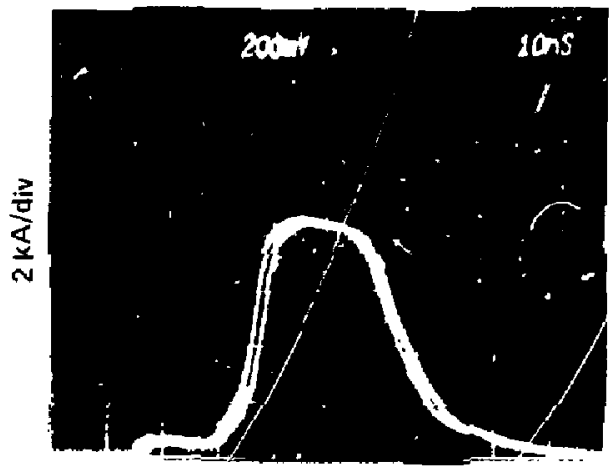

$10 \mathrm{~ns} / \mathrm{div}$

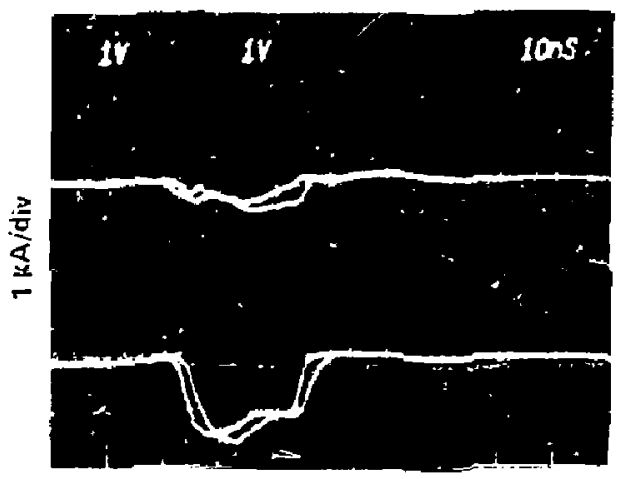

$10 \mathrm{~ns} / \mathrm{div}$

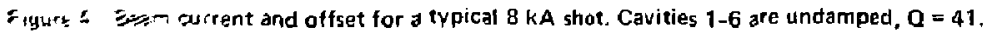

and 7 and 8 are damped, $Q=7$. This shot is compared with code runs and is labeled experiment no. 1 . 


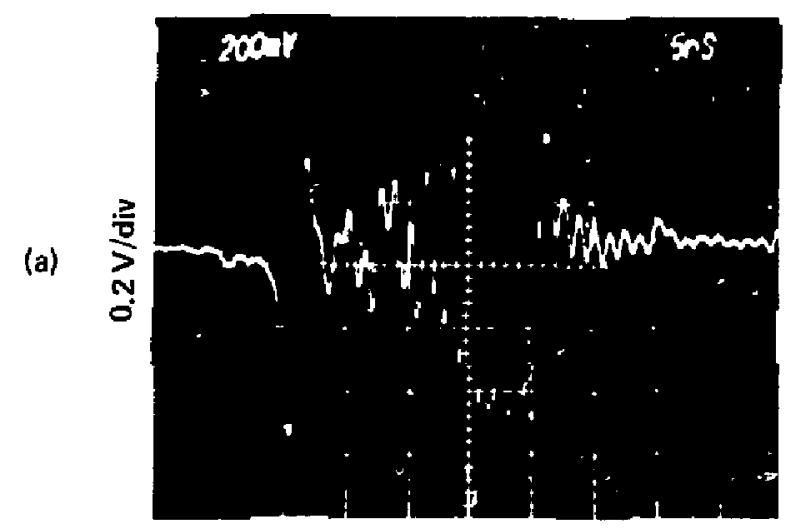

$5 \mathrm{~ns} / \mathrm{div}$

(b)

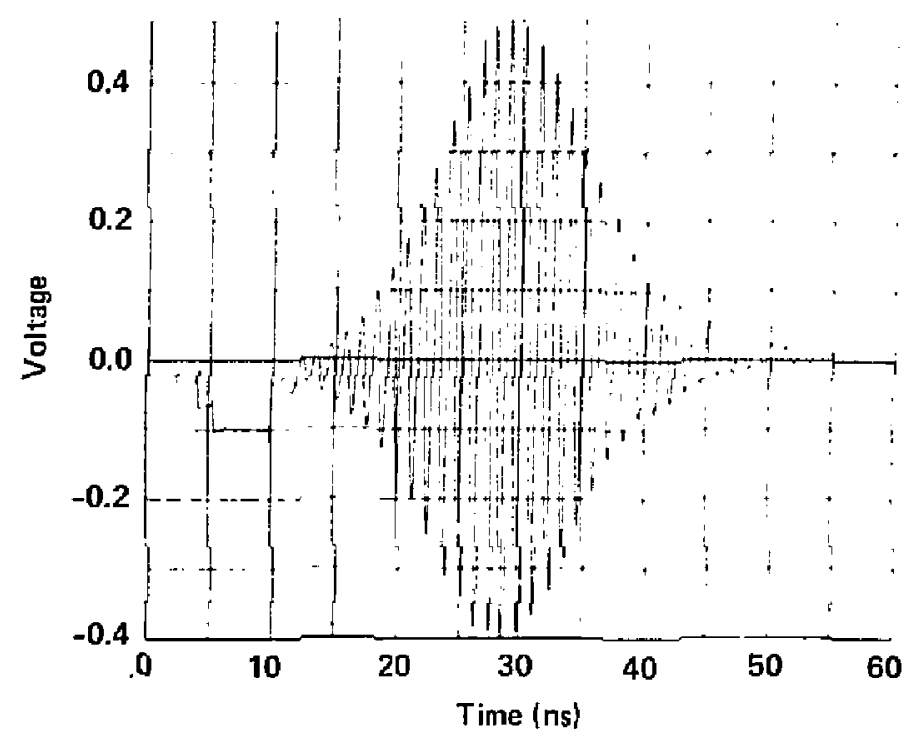

Figure 5. Comparison of the if loop signal at the outputof the accelerator with the code result for experiment no. 1. (al rf loap signal, attenuated 500 times.

(b) Code output based on the input current and offset profile of Figure 4, also attenuated 500 times. 
(a)

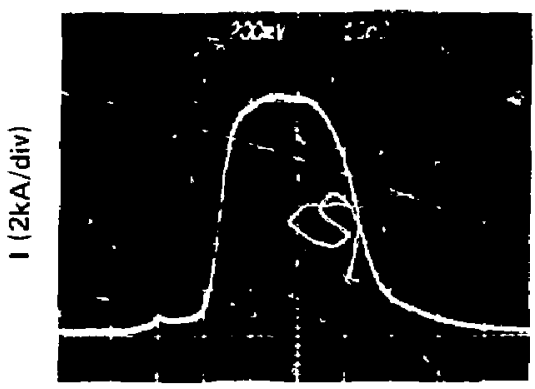

$10 \mathrm{~ns} / \mathrm{div}$

(b)

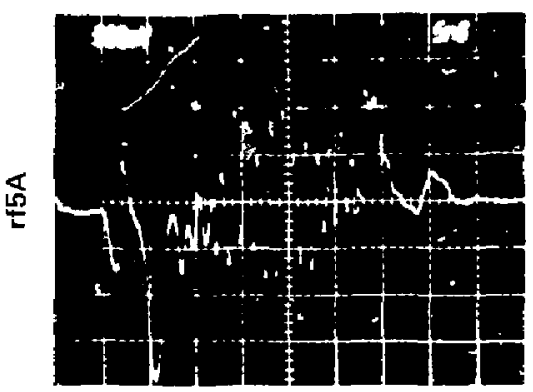

$5 \mathrm{~ns} / \mathrm{div}$

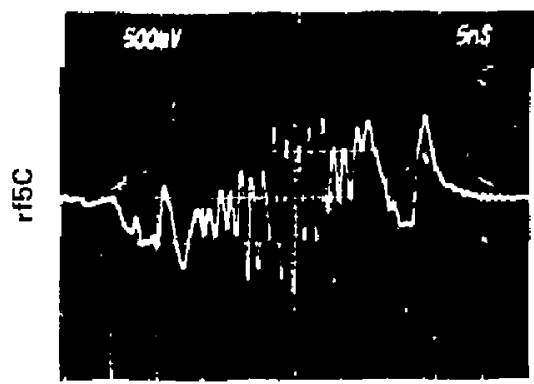

$5 \mathrm{~ns} / \mathrm{div}$

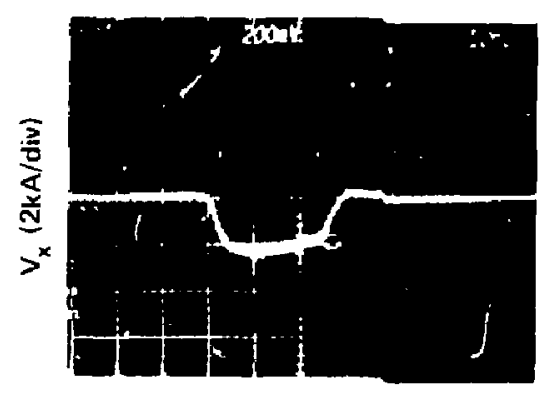

$10 \mathrm{~ns} / \mathrm{div}$

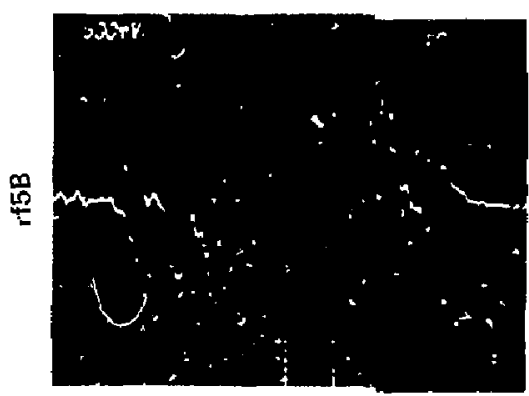

$5 \mathrm{~ns} / \mathrm{div}$

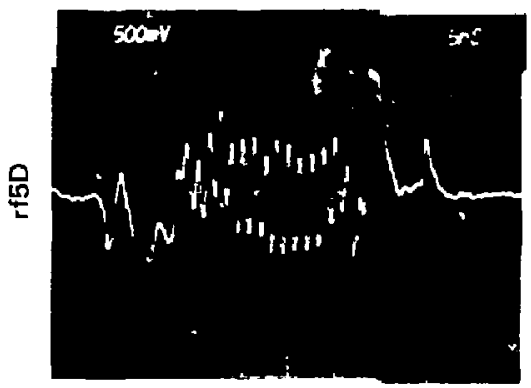

5 ns/div

Figure 6. Experiment no. 2. A $10 \mathrm{kA}$ shot with a large if output. All eight cavities are damped, $Q=7$.

(a) Beam current and $x$ offset. The $y$ offset was observed to be much smaller and is omittey.

(b) if loop signals of the four quadrants at the output of the accelerator. Vertical scales are $0.5 \mathrm{~V} / \mathrm{djv}$, with the signals attenuated 100 times. 
(a)

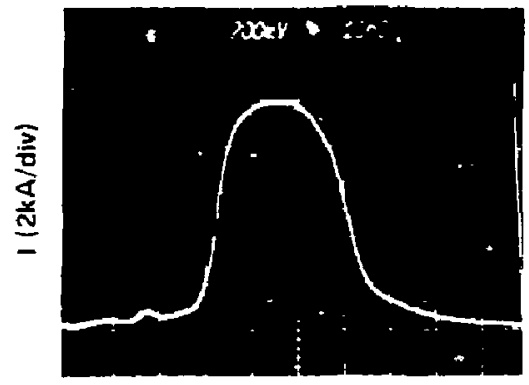

$10 \mathrm{~ns} / \mathrm{div}$

(b)

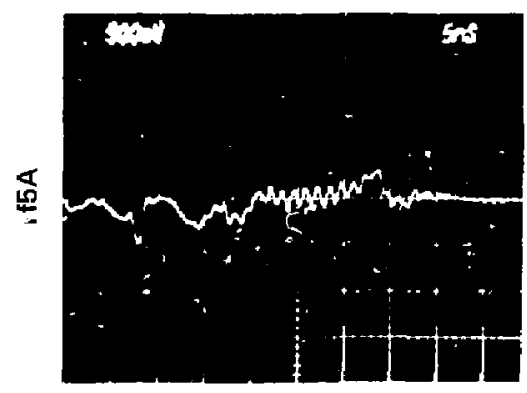

$5 \mathrm{~ns} / \mathrm{div}$

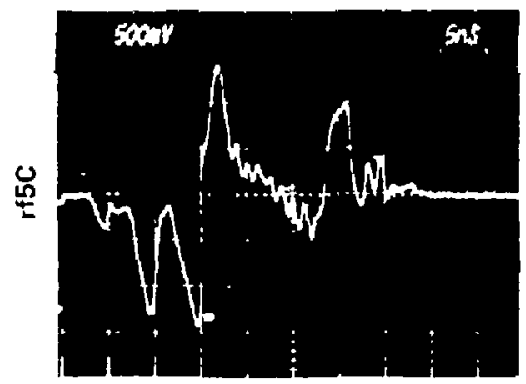

$5 \mathrm{~ns} / \mathrm{div}$

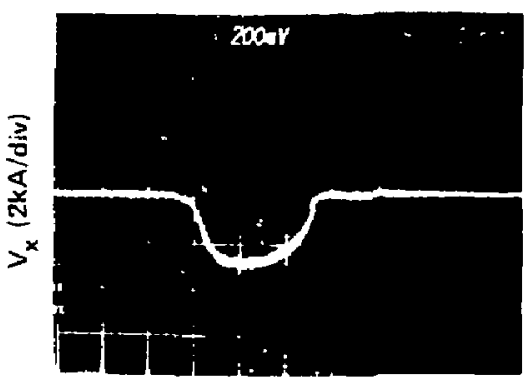

$10 \mathrm{~ns} / \mathrm{div}$

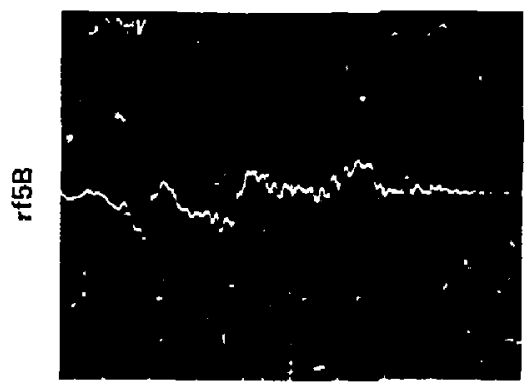

$5 \mathrm{~ns} / \mathrm{div}$

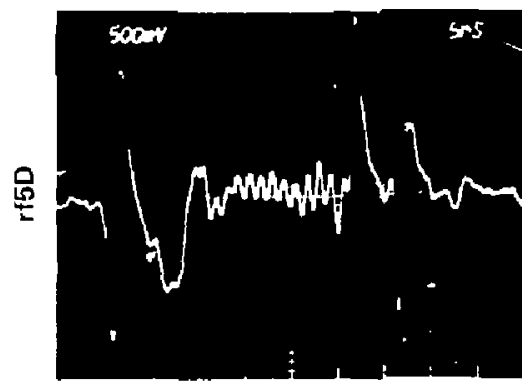

5 ns/div

Figure 7. Experiment no. 3. A $10 \mathrm{kA}$ shot with a small if output. Same parameters and correspondind photographs as in Figure 6. 


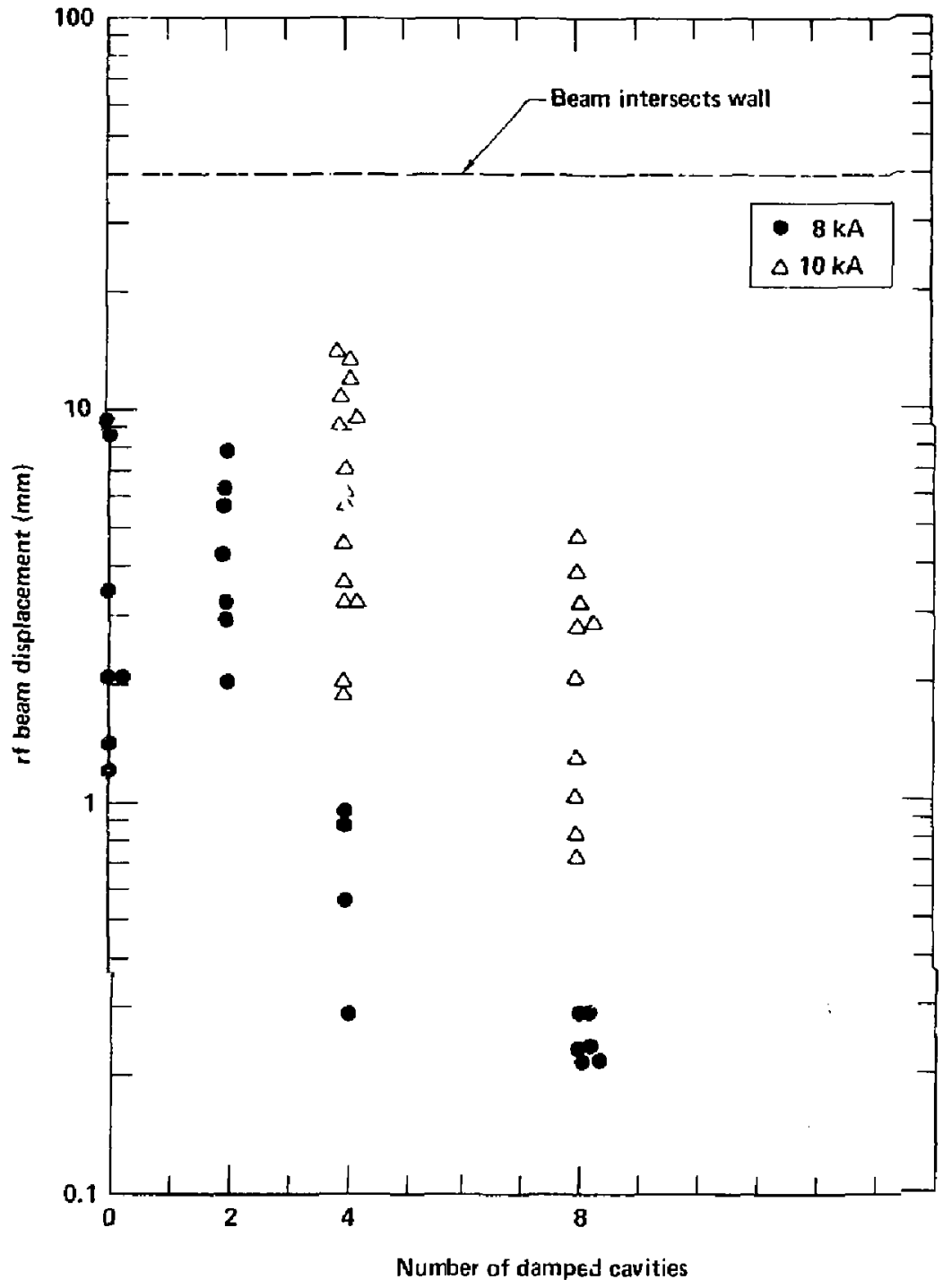

Figure 8. Amplitude of the BBU oscillations of the ETA beam as a function of the number of daniped accelerator cavities at both 8 and $10 \mathrm{kA}$. Each point is one shot. The spread in amplitudes is due to pulse to pulse variation in beam current, current risetime and beam offset. 


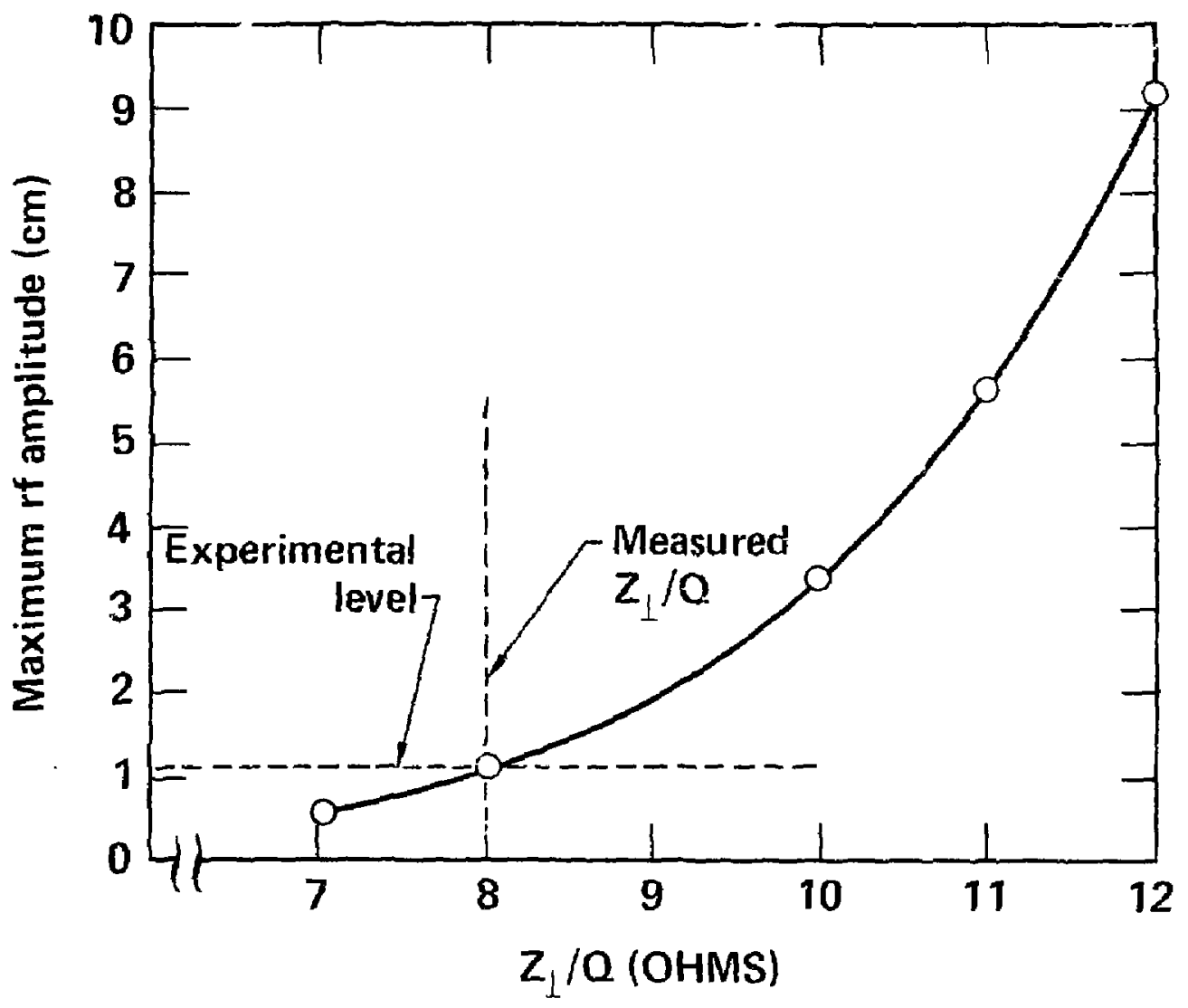

Figure 9. The sensitivity of the calculated if amplitude to $Z, Q$ for Experiment \#1. Cavities 1-6 were undamped with $Q=41$ while cavities 7 and 8 were damped with $Q^{\prime}$ 's of 7 . The peak beam current was $8 \mathrm{kA}$. 


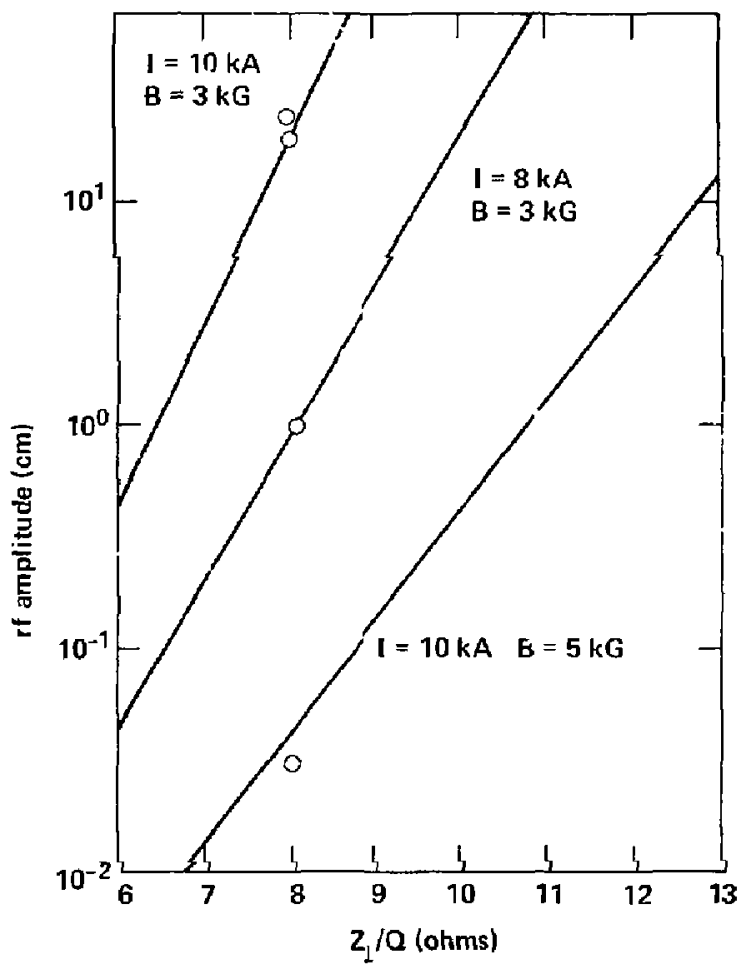

Figure 10. ATA beam breakup predictions for a variety of operating conditions as a function of $Z_{1} / 0$. The final if amplitude in $\mathrm{cm}$ is plotted againt $Z_{1} / Q$ in ohms. All curves are for $Q=5, d_{0}=0.1 \mathrm{~cm}$ and a current rise time of $10 \mathrm{~ns}$. The bearn current and solunoidal magnetic field strength are shown on the curves. 


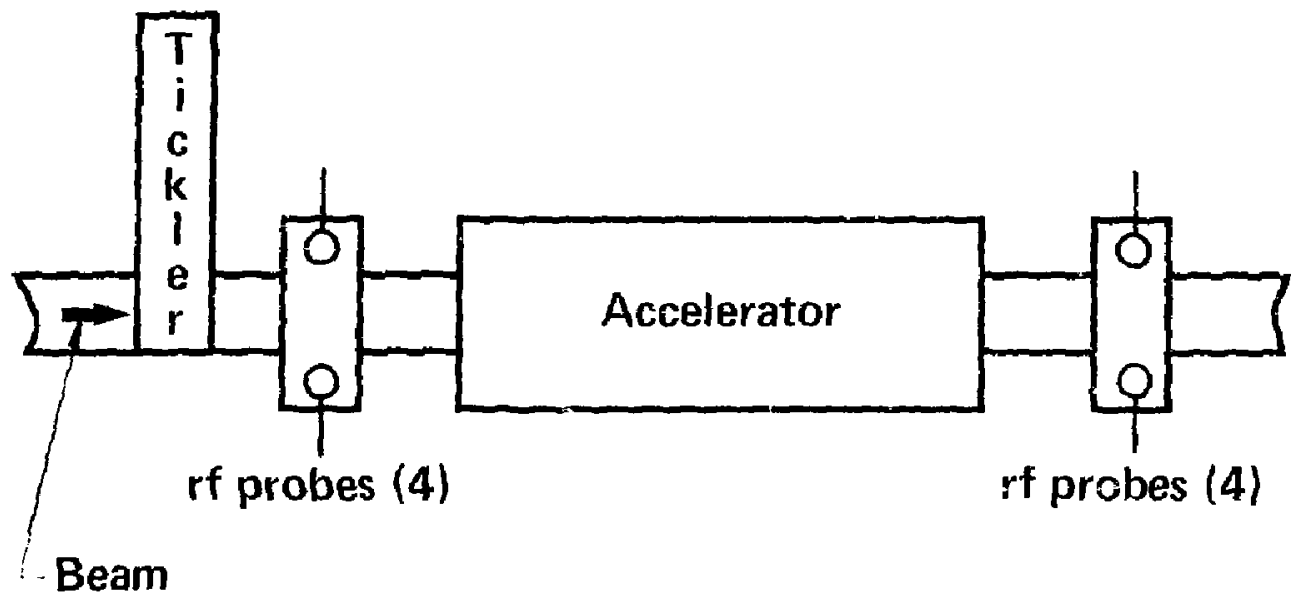

Figure 11. Schematic representation of proposed tickler experiment. A self-excited, tunable of cavity is inserted into the ETA beamline upstream of the accelerator. $4 \mathrm{rf}$ loops monitor the transverse oscillations impressed on the beam by the tickler cavity. The growth of these transverse oscillations is measured at the output of the accelerator by 4 additional if loops. 